\title{
BMJ Open Role of community drug and alcohol services in physical healthcare for people who use illicit opioids: a qualitative study of clinical staff in the UK
}

To cite: Bradbury M, Lewer D. Role of community drug and alcohol services in physical healthcare for people who use illicit opioids: a qualitative study of clinical staff in the UK. BMJ Open 2021;11:e046577. doi:10.1136/ bmjopen-2020-046577

- Prepublication history for this paper is available online. To view these files, please visit the journal online (http://dx.doi. org/10.1136/bmjopen-2020046577).

Received 04 November 2020 Accepted 13 July 2021

Check for updates

(C) Author(s) (or their employer(s)) 2021. Re-use permitted under CC BY-NC. No commercial re-use. See rights and permissions. Published by BMJ.

${ }^{1}$ Institute of Epidemiology and Healthcare, University College London, London, UK

${ }^{2}$ Plymouth University Peninsula School of Medicine, Plymouth, UK

Correspondence to

Dan Lewer; d.lewer@ucl.ac.uk

\section{ABSTRACT}

Objectives To understand how clinicians working in addiction services perceive their responsibilities for physical healthcare of clients who use opioids, and how physical healthcare could be improved for this group. Design Qualitative study comprising semistructured interviews.

Participants 16 clinicians, including nurses and nurse practitioners, nurse consultants, addiction psychiatrists, specialist general practitioners and psychiatry specialty registrars.

Setting Community-based drug and alcohol treatment services in the UK, with services including outpatient opioid agonist therapy.

Results We identified three overarching themes. First, clients have unmet physical health needs that are often first identified in community drug and alcohol services. Participants reported attempts to improve their clients' access to healthcare by liaising directly with health services and undertaking other forms of health advocacy, but report limited success, with many referrals ending in non-attendance. Second, most participants saw their role as supporting access to mainstream health services rather than providing physical healthcare directly, though sometimes reported frustration at being unable to provide certain treatments such as antibiotics for a respiratory infection. A minority of participants felt that people who use illicit opioids would be best served by an integrated 'one-stop-shop' model, but felt this model is currently unlikely to receive funding. Third, participants felt isolated from other health services, in part due to commissioning arrangements in which funding is provided through local government rather than the National Health Service.

Conclusions Clinicians participating in this study serve a patient group with unmet physical health needs, but lack the resources to respond effectively to these needs.

\section{INTRODUCTION}

Use of illicit opioids such as heroin is associated with poor health and unmet healthcare needs, and mortality rates are several times the general population. ${ }^{12}$ Although research and interventions focus on prevention and treatment of drug overdoses and viral infections, cohort studies in highincome countries suggest that more excess

\section{Strengths and limitations of this study}

To our knowledge, this is the first study examining the role of specialist community drug and alcohol services in the provision of physical healthcare. This in-depth qualitative study examines clinicians' perceptions about the role of these services in the wider healthcare system, and how barriers to better physical healthcare might be overcome.

- We interviewed clinicians from a diverse range of backgrounds, including those from different grades and professional groups, from geographical areas across the UK, and from different types of provider.

- Given the differing approaches to drug treatment services and general healthcare internationally, the results are mostly of interest within the UK.

The study does not include the perceptions of clients of community drug and alcohol services.

deaths in this population are caused by long-term physical health problems such as cardiovascular disease, cancers and respiratory disease. ${ }^{34}$ Poor physical health relates to the direct effects of drugs, lifestyle factors such as tobacco smoking and poor diet, and social exclusion such as homelessness and imprisonment. ${ }^{5}$ The importance of physical comorbidity is increasing as the population of people who use illicit opioids gets older. In 2019/2020, among people starting treatment for illicit opioid use in England, $48 \%$ were aged 40 or older, compared with $29 \%$ in 2012/2013. ${ }^{67}$

Despite the high need, qualitative research suggests that access to healthcare is poor. Barriers reported by people who use illicit opioids include: (A) judgemental and stigmatising attitudes among healthcare professionals; (B) competing priorities such as the need to find money, accommodation or drugs; (C) difficulty with transport or timekeeping; (D) being barred from services following missed appointments, or because some services will not treat patients with drug 
dependence; and (E) healthcare professionals ascribing symptoms to the use of drugs ('diagnostic overshadowing'). ${ }^{8-10}$ Perceptions reported by healthcare professionals include: (A) insufficient knowledge and training to treat this patient group, particularly with regard to pain relief; (B) difficulty meeting the high needs of this patient group within already high workloads; $(\mathrm{C})$ that people who use drugs seek prescriptions for non-medical purposes. $^{1112}$

Community drug and alcohol services (CDAS) provide an important point of contact between people who use illicit opioids and the healthcare system. In March 2020, a total of 140599 people were enrolled in treatment for opioid use in services in England, making up 52\% of all clients in treatment for drug or alcohol use. ${ }^{6}$ The number of people who use illicit opioids and who are not in treatment is unknown, but estimates suggest that approximately half are currently enrolled. ${ }^{13}$ The history of these services is described in more detail elsewhere. ${ }^{14}$ Briefly, services expanded in the 1990s and early 2000s under a National Health Service (NHS) body called the National Treatment Agency. From 2013, commissioning was moved to local government. This coincided with financial cuts in many local areas, more frequent recommissioning, greater diversity among providers with more charities delivering clinical treatment and a shift of focus from retaining clients in treatment towards recovery. This change of focus included the use of "payment for performance' schemes in which CDAS received payments for discharging patients who are recorded as abstinent from drugs. 15

In the UK, CDAS primarily aim to help their clients to stop or reduce their use of alcohol and drugs through psychosocial interventions and medicines such as methadone and buprenorphine (for opioid dependence). They may also offer ancillary health services, in recognition that some clients have unmet health needs. Emerging evidence suggests that some CDAS in the UK have developed models including 'in-reach', in which specialists provide visiting clinics, with recent evidence focusing on respiratory care and hepatitis $\mathrm{C}$ treatment. ${ }^{16-19}$ Current UK guidelines (known colloquially as the 'Orange Book') recognise the increasing health needs of clients, promote joint working with other health services and say that CDAS should undertake a range of general health assessments and tests, with referrals to general practitioners (GP) and secondary care as needed. ${ }^{20}$ However, it is unclear whether these actions are being done.

We aimed to explore how clinical staff in CDAS identify and address physical health problems among clients who use illicit opioids, what they perceive to be their responsibilities in terms of physical health and how they think that physical healthcare could be improved for these clients.

\section{METHODS}

We conducted a qualitative study including semistructured one-to-one interviews with clinicians working in
CDAS in the UK, and thematic analysis of transcribed interviews.

\section{Setting and participants}

We used purposive sampling, which selects participants who are especially knowledgeable or experienced in the area of interest, based on predefined criteria. ${ }^{21}$ Inclusion criteria were that participants: (1) were qualified doctors or nurses; (2) currently work in a communitybased drug and alcohol treatment service in the UK, including provision of opioid agonist therapy (methadone and buprenorphine); and (3) have at least 3 years of clinical experience. We focused on clinically qualified staff because they have expertise in health needs of their clients and in the design of health services. Potential interviewees were identified through regional policy forums, provider networks and snowball sampling (where an interviewee suggests another interviewee). People had the opportunity to ask researchers about the study prior to participation, and provided written consent. We aimed to include participants from a mixture of roles, geographical regions and provider types (including those delivered by NHS trusts and independent charities). The recruitment period was May to December 2019.

\section{Data collection}

Participants were offered face-to-face or telephone interviews according to their preference. Interviews were undertaken with either MB or DL. We developed a topic guide prior to the first interview, with topics including the participants' perceptions of: their clients' health and healthcare access; their role in terms of physical healthcare; barriers and enablers to better physical healthcare for their clients; and 'ideal' models of physical healthcare for their clients. We allowed participants to discuss other topics that they felt were relevant, and updated the topic guide several times during data collection as we identified new themes. Interviews lasted between 16 and $76 \mathrm{~min}$ with the median of $34 \mathrm{~min}$.

\section{Transcription, data management and analysis}

Interviews were digitally recorded and transcribed verbatim. We used field notes taken immediately after interviews to identify emerging themes. For example, some participants discussed their relationship to other health services and professions, and we therefore asked about the importance of these relationships in subsequent interviews. After interviews were completed, we analysed transcripts using the principles of thematic analysis, following the process described by Braun and Clarke (see table 1). ${ }^{22}$ Transcripts were read several times and analysed using an inductive 'open coding' process to create 293 codes using NVivo V.12. Half of the interviews were coded by one author and half by another, with two interviews double coded and discussed between authors to check for reliability in the coding approach. Codes were deduplicated and grouped into a coding framework, which was used to identify the most important themes, 
Table 1 Summary of qualitative analysis and development of themes

\begin{tabular}{|c|c|}
\hline Analysis stage & Description \\
\hline Familiarisation & $\begin{array}{l}\text { Transcription of interview audio files, } \\
\text { reading and re-reading of transcripts. }\end{array}$ \\
\hline $\begin{array}{l}\text { Generating } \\
\text { initial codes }\end{array}$ & $\begin{array}{l}293 \text { codes generated by inductive 'open } \\
\text { coding', with two transcripts double coded } \\
\text { and discussed between authors. }\end{array}$ \\
\hline $\begin{array}{l}\text { Searching for } \\
\text { themes }\end{array}$ & $\begin{array}{l}\text { Collaborative grouping of codes into } \\
\text { similar topics. }\end{array}$ \\
\hline $\begin{array}{l}\text { Reviewing } \\
\text { themes }\end{array}$ & $\begin{array}{l}\text { Identification of initial themes, which were } \\
\text { mapped to a sample of five transcripts } \\
\text { to ensure they were representative of the } \\
\text { data. }\end{array}$ \\
\hline $\begin{array}{l}\text { Defining and } \\
\text { naming themes }\end{array}$ & $\begin{array}{l}\text { Authors discussed and refined themes, } \\
\text { and selected key themes. }\end{array}$ \\
\hline $\begin{array}{l}\text { Producing the } \\
\text { report }\end{array}$ & $\begin{array}{l}\text { Description of themes illustrated with key } \\
\text { quotes identified throughout the process. } \\
\text { The draft report was sent to a subset } \\
\text { of participants for feedback ('member } \\
\text { checking'). }\end{array}$ \\
\hline
\end{tabular}

perceptions that were consistent across participants and areas where participants had different perceptions.

\section{Patient and public involvement}

The need for the study emerged from DL's engagement with clients and staff at CDAS in London, as part of a wider research programme. This engagement included focus groups and one-to-one discussions. Clients reported challenges accessing health services such as GPs, supporting the findings of existing qualitative research. Staff were aware of these challenges, and worked with clients or implemented schemes to try and improve the situation. These experiences had not been documented previously.

\section{FINDINGS}

\section{Participants}

A total of 17 individuals agreed to participate, including 4 consultant psychiatrists, 4 nurses or nurse practitioners, 1 nurse consultant, 5 GPs with a special interest in addiction medicine, 2 psychiatry specialty trainees and 1 drug worker from a nursing background. Nine participants were in London and the South-East, 3 in South-West England, 1 in North-West England, 1 in Wales and 3 in Scotland. Two participants were employed by the same service and some were employed by multiple services, and in total we captured 17 separate CDAS. Thirteen services were run by the NHS and four by charities. One participant was not currently employed by an eligible service and was excluded from analysis, leaving 16 transcripts for analysis. There were no other withdrawals from the study. The characteristics of these 16 participants are summarised in table 2. Eleven interviews were conducted face to face and five were conducted by phone.

\begin{tabular}{llr}
\hline Table 2 & Participant characteristics & \\
\hline Variable & Level & $\mathbf{n}$ \\
\hline Total & & 16 \\
Professional group & Consultant psychiatrist & 4 \\
& Nurse consultant & 1 \\
& GP with special interest in & 4 \\
& addiction & \\
& Nurse practitioner & 4 \\
& Specialty registrar (resident) & 2 \\
& Drug worker with nursing & 1 \\
& background & \\
Geographical region & London and the South-East & 9 \\
& South-West & 2 \\
& North-West & 1 \\
& Wales & 1 \\
& Scotland & 3 \\
Years of experience & 5 years or less \\
in CDAS & More than 5 years & 4 \\
Sex & Male & 12 \\
& Female & 6 \\
& & 10 \\
\hline
\end{tabular}

CDAS, community drug and alcohol services; GP, general practitioner.

We identified three overarching themes: (1) physical health needs are often first identified in CDAS and clinicians act as health advocates; (2) clinicians feel they should support healthcare access, rather than provide integrated care; and (3) clinicians feel isolated from other health services.

\section{Theme 1: physical health needs are often first identified in CDAS and clinicians act as health advocates}

All participants discussed the health needs of their clients in detail, and said that their health is typically worse than people of the same age in the general population. In particular, several participants discussed the high prevalence of chronic respiratory problems and the lack of diagnosis and treatment. Participants said that most clients are unlikely to seek help for symptoms of physical health problems; often do not attend healthcare appointments; have difficulty getting diagnoses; and often have poor access to treatment pathways for long-term conditions. Some participants also discussed the variation in healthcare access across clients:

We have got some service users who are very well engaged with primary care and attend regular diabetes reviews or asthma reviews or COPD reviews ... Equally we have a larger majority of people whose health is quite poor and they don't engage with those services. (Nurse consultant)

When asked where their clients seek medical care, participants said that the CDAS (ie, their own service) 
and hospital accident and emergency are often the first points of contact. Participants said that clients approach CDAS for help because they are open access and nonjudgemental. They emphasised the importance of building trust with their clients over a period of time, and not cancelling appointments or otherwise penalising clients who do not attend. All participants described extensive barriers to healthcare for their clients, particularly relating to stigma, with one participant saying that 'GPs just put up barriers because they think the main reason they come is to get hold of drugs' (GP 1). Participants felt that the approach in CDAS contrasted with this:

We are here, open-door policy, friendly, no barriers. They will come again and again for physical problems. It is then up to us to get them to see the GP or to get them to go to the hospital. (Consultant psychiatrist 2)

On identifying a physical health problem, participants would recommend that clients visit their GP, or help them make appointments, but reported that clients often did not attend:

You can sign-post people to GPs but they don't then go. Or they actually find it really hard to get appointments. So we tend to do quite a bit of ringing the surgery and booking in appointments. But that doesn't guarantee they'll go. (Drug worker)

Most participants expressed a duty to act as health advocates for their clients. The most common examples were helping clients book GP and outpatient appointments, reading and responding to letters and encouraging other health services to see clients at a time when the client is more likely to attend (sometimes immediately). Other examples included peer support, in which emotional and practical support is provided by trained volunteers with personal experience of using opioids, and when staff at CDAS play a coordinating role in intensive healthcare interventions:

People end up in a really bad way and everyone is really worried. An example being when they have tuberculosis or they have endocarditis ... All of a sudden specialist services realise the key to this person is the substance misuse service, because that's the place they actually attend. (GP 1)

Some elements of physical healthcare were unanimously considered 'core' to CDAS. All participants described physical health assessments for new clients and at variable intervals, and screening for bloodborne viruses (though the viruses screened varied, with all services screening for hepatitis $\mathrm{C}$ and some also screening for hepatitis B and HIV). The physical health assessments were often focused on the safety of opioid agonist medications:

It happens around the addiction if you see what I mean. It is all focused on whether they are well enough to have Subutex or methadone ... It's not about wider health issues. I mean you'd probably struggle to get them to do anything about an infected leg ulcer for example ... It's not their area of expertise, it's not an area they are comfortable with. (Nurse practitioner 2)

\section{Theme 2: clinicians feel they should support healthcare access, rather than provide integrated care}

All participants agreed that the best physical healthcare is available in general practice and hospitals. Related to the health advocacy role described above, participants saw their role in terms of physical health as supporting access to mainstream health services. Participants gave three reasons for this.

First, medical expertise in CDAS was primarily from a psychiatry background:

I'm a psychiatrist. I'm not a general practitioner. I'm way out of practice for general health, I can't do that. Apart from checking pulse and blood pressure. I'm not able to do much more than that. (Consultant psychiatrist 4)

The complexity of clients' health needs, particularly related to liver disease, respiratory disease and mental health problems, meant that specialist skills are often needed, beyond what could be delivered within CDAS:

As soon as someone's liver starts failing that becomes much more complex. Anyone who is not having treatment for their HIV, the potential illness that they can have are not going to be easy to manage. So it makes sense to make sure they are supported in the specialist services. And that's what the focus is, to get them into the right services. (Nurse practitioner 2)

Second, some participants discussed their clients' right to the same health services as the general population. They said that clients want to be treated like everyone else and should have the opportunity to build relationships with their GP. One participant said colocating healthcare and drug and alcohol services means 'telling that person that you're in addiction services, and you're different' (GP 2). Some participants felt that engaging with health services is part of clients' recovery and enables them to develop personal and social resources, and provision of 'one-stop-shop'-type healthcare may impede recovery:

Initially it seemed odd to me that we couldn't do that coincidental stuff. So you turn up for your substance misuse appointment but you've run out of your asthma inhalers. Now a GP, if you turned up with your infected toenail but said can you check my [blood pressure], most GPs would see that as an opportunity ... It was initially quite difficult for me to get my head around that I couldn't do that stuff ... But I think if more of their medical care starts getting delivered from the substance misuse services to some extent we are further isolating them from normal care streams. We're not enabling them to make use of all of the 
services that are provided for them ... Trying to support the client into accessing regular services is probably more sustainable than simply abandoning ship and delivering everything to them in an institutional way. (GP 1)

Third, participants discussed constrained financial resources and the need to focus on providing treatment for drug dependence. They felt that additional responsibilities would not be safe or desirable and would duplicate other services. One participant discussed cuts over recent years and the difficulty of providing broader care:

You have to figure out ways of focusing on the core of your business, and you need to define that, you don't have a lot of time for additional stuff ... Unless there is an injection of cash, which is very very unlikely, we are going to end up neglecting our core business to do something that someone else is supposed to be doing. (Consultant psychiatrist 3)

Despite their efforts to improve access to mainstream healthcare, all participants acknowledged that many of their clients have poor access. In contrast to the participants who felt that CDAS should focus on supporting better access to mainstream health services, a minority of participants felt that CDAS should provide a 'one-stopshop' service in which primary care is provided alongside drug treatment. These participants often focused on the needs of clients with the greatest barriers to healthcare, including those experiencing homelessness, using a lot of illicit drugs, with serious mental health problems and with no recourse to public funds. For example, one participant discussed the benefits of a previous CDAS model that offered GP appointments and wound care:

We have a lady who is coming here who had a DVT. I could see it's flaring up. She came in and there was an ulcer which looked bad. Each time she comes in it was can you please see your GP ... I used to work in a service that had a GP clinic in there. That was really good ... People who had ulcers could have their wound dressed. They come for the drug service but they get everything in there. (Nurse practitioner 1)

Although the majority of participants felt that colocated primary care and drug treatment is not desirable, most nonetheless described existing models in which some elements of physical healthcare are provided at the CDAS in partnership with other local health services. This usually involved hepatitis $\mathrm{C}$ treatment, as part of the hepatitis C elimination programme in the UK. Some participants described visiting specialists running clinics within CDAS, which was described as 'in-reach', while others were directly commissioned to deliver hepatitis $\mathrm{C}$ treatment. Other participants described hospital outpatient services that reserved clinics for their clients, with CDAS staff responsible for booking appointments. One participant described this model for respiratory and gastroenterology clinics. These models were felt to improve accessibility, but were often temporary because they relied on specific individuals or short-term project funding.

In terms of medicines, most participants were only able to prescribe opioid agonists, and some expressed the desire to prescribe a wider range of medicines in an opportunistic manner. Some were able to prescribe specific medications through Patient Group Directives (template prescriptions used in specific circumstances), with participants mentioning antibiotics for skin and respiratory infections, vaccinations and long-acting reversible contraception. Perceptions about broader prescribing were mixed. One participant wanted to make simple prescriptions where they were needed, and said commissioners determined the limited prescribing options. Others felt that a more general prescribing function would be undesirable because it would demand more clinical skills, may duplicate primary care and could change their relationship with clients. GPs, for example, sometimes have difficulty negotiating prescriptions such as gabapentinoids and benzodiazepines; drugs that are often used alongside illicit opioids.

\section{Theme 3: clinicians feel isolated from health services}

All participants felt their services were comparatively poorly funded, and some felt their funding was insufficient to provide good quality core services. Participants related cuts to their funding to the Health and Social Care Act 2012, which led to CDAS being funded through local government rather than the NHS. Participants said that these cuts led to reduced staffing and a more limited scope of services and reduced clinical staffing:

The previous service I used to work in, we had nurses that worked in our needle exchange service. They did full leg ulcer treatment. They did full dressings. And would see any of our clients across the service ... I haven't recently seen quite so much of that, simply because I think most services around the county are struggling to deliver on what budget you're given. (Nurse practitioner 4)

Many participants expressed frustration about cuts, describing them as counterproductive because they would lead to greater healthcare use in other parts of the healthcare system. Some participants felt that cuts were related to stigma towards people who use illicit drugs, with a perception that austerity in the UK had been disproportionately applied to CDAS. Participants were pessimistic about the prospects for better funding:

The less you support people the less you help people in that situation. They become more chaotic and the more chaos means that they access services in a way that's more expensive. It's a bit counterproductive. But yeah. That's a political question. (Nurse practitioner 2)

As well as reducing funding, participants described short 'commissioning cycles' as a barrier to investment 
and development of relationships with other health services:

Why would I embark on a long and painful process of meeting with all kinds of people and writing policies and buying hardware and equipment and getting remote logins if we lose the contract next year and it will all just get scrapped? (GP 1)

Participants discussed feelings of isolation from other health services. Fragmented approaches to commissioning mean that CDAS are not able to provide holistic care for their patients, and can only refer patients to other health services. Referring to commissioning via local authorities, one participant said:

That happened back in 2012 with the Health and Social Care Act. But it means we are only really allowed to prescribe opioid substitution medications, that's the only thing on our license ... There's a lot of 'not out of my budget' approaches. The commissioner has decided that's what we can prescribe so that's it. So sometimes we see people and they need antibiotics, or they need an inhaler refill ... And we can just refer them to the GP. And more often than not they don't go. (Psychiatry specialty registrar 1)

Most participants felt their communication with other health services was poor, describing difficulty finding out about interactions between clients and other health services. All participants felt that communication with GPs enabled better drug treatment and general physical healthcare, but most felt that this communication was partial and varied by GP surgery. Some participants, particularly those working in services not provided by the NHS, reported that these difficulties were due to incompatible computer systems or a lack of data-sharing protocols.

\section{DISCUSSION}

\section{Summary}

Participants in this study felt that their clients have extensive unmet physical health needs. They reported that CDAS are sometimes the only point of contact that clients have with health services. They felt isolated from other health services and had limited resources to support clients with physical health problems.

Although all participants recognised these problems, they had different perceptions of the appropriate role of CDAS in the provision of physical healthcare. Most felt that CDAS are not an appropriate place for physical healthcare to be delivered. Many nonetheless provided some elements of physical healthcare. The most common example was treatment for hepatitis $\mathrm{C}$, with other examples including wound care, contraception and tetanus vaccinations. Many participants reported difficulty providing interventions deemed essential in clinical guidelines, ${ }^{20}$ citing disinvestment in CDAS. Overall, the provision of interventions targeted at physical health varied widely among our participants. Participants felt that longer term management of physical health problems is best done in primary care, and therefore focused on making referrals and advocating for clients. This approach had limited success, and referrals to GPs and other health services often end in non-attendance.

Most participants were sceptical about colocated models (or 'one stop shops') of primary care and drug treatment services. This scepticism was based on concerns about funding, equitable care and the ability of CDAS to deliver ancillary services in the context of financial cuts and reduced staff. While participants did not articulate a common solution or preferred model of physical healthcare for their clients, all discussed the need for more financial resources, more staff and better integration with other health services. 'Integration' has many meanings. For example, participants discussed models including 'in-reach' where specialists visit CDAS, dedicated clinics for CDAS clients at hospitals and more multidisciplinary services. Clearer definitions of the possible models of integration may help professionals and clients to advocate for solutions.

\section{Strengths and limitations}

To our knowledge, this is the first study exploring staff perceptions of the role of CDAS in the provision of physical healthcare. Our sample included participants from different clinical backgrounds and grades, different types of service provider (with a mixture of NHS and charity organisations) and different regions of the UK.

Despite this variation within the sample, our participants may differ from other clinicians working in CDAS. First, participants may be particularly engaged with physical health problems because we recruited through policy forums and networks where the general health of people who use drugs is discussed. Second, several participants worked in urban areas such as London, Glasgow and Cardiff, where there are clusters of specialist health services for marginalised populations. Participants working in these areas described the benefits of referring clients to open-access health services designed for people experiencing homelessness, for example. For these reasons, clients of participants in our study may have better-than-average access to physical healthcare. Third, the small sample size means we may have selected an unusual group even if we used random sampling.

Given the sampling method and our qualitative methodology, our findings cannot be generalised to other clinicians or services.

To keep the study focused, we only included CDAS staff with clinical training. This means we excluded other groups with expert knowledge about this topic, particularly non-clinical CDAS staff such as drug or social workers, and clients. Exclusion of clients means that the barriers to healthcare are reported 'second-hand'. However, other studies have documented the healthcare experiences of people who use illicit drugs. ${ }^{8-10}$ 


\section{Comparison with existing literature}

A body of qualitative research has documented barriers to healthcare for people who use opioids. ${ }^{9-12} 23$ These barriers are consistent with those reported by participants in our study, though participants in our study placed greater emphasis on individual behaviours, while studies that include people who use opioids emphasise structural barriers such as stigma. Participants discussed common experiences of social exclusion in this population, such as homelessness, prison, serious mental health problems and sex work, which is consistent with other descriptions of people who use illicit opioids. ${ }^{35}{ }^{24}$ Other research suggests that one in three people in opioid agonist therapy has chronic obstructive pulmonary disease ${ }^{16}{ }^{17}$ consistent with participants' concern about the respiratory health of their clients. The importance of these issues is likely to increase in the future. In the UK, large numbers of people started using heroin in the 1990s..$^{25}$ As this cohort ages, the average age of CDAS clients is increasing, and the health and social needs of this group are becoming more complex.

Participants had different opinions about the desirability of a 'one-stop-shop' approach to healthcare for people who use illicit opioids. Those who did not support this model felt that it is unfeasible, or that it conflicts with patient recovery. We are not aware of other studies that have shown this tension between patient recovery and colocation of services. 'One-stop-shop' models have been developed for populations including people who use drugs, living with HIV and experiencing homelessness. ${ }^{26-29}$ Research in the USA has evaluated integrated drug treatment and primary care (sometimes called 'shared care'). ${ }^{30} 31$ These studies suggest that the model is associated with increased drug abstinence, but the association with health outcomes is unclear, partly relating to the difficulties with study design. Some participants in our study discussed the importance of offering additional support when clients use open-access harm reduction services such as needle exchanges, as some individuals may not use any other services. This perspective reflects the approach often taken in drug consumption rooms, which first offer food, clothes and basic acute medical care such as abscess and wound care, and later offer referrals to health services that can provide longer term management of health conditions. ${ }^{32}$ In this model, the harm reduction service is the 'way into' other health services. This reflects the 'health advocacy' role that participants discussed, though participants did not feel they have the resources to play this role effectively.

All participants in our study discussed financial cuts to their services. Cuts are difficult to measure but were estimated at 30\% on average between 2012 and 2015, with further cuts planned. ${ }^{33}$ These cuts were highlighted in a recent government-sponsored review, ${ }^{34}$ which said that 'a prolonged shortage of funding has resulted in a loss of skills, expertise and capacity from this sector', that it is likely 'many areas are now offering the bare minimum service with large increases in worker caseloads' and that cuts have resulted "in what are seen as "nice-to-haves" being cut'. These 'nice-to-haves' are likely to include interventions that aim to improve access to physical healthcare.

\section{CONCLUSIONS AND IMPLICATIONS FOR RESEARCH}

Our study adds to existing evidence that people who use opioids have unmet health needs. We found that CDAS recognise this problem and act as health advocates for their clients, but report limited success. Participants did not articulate a common solution, discussing potential improvements to the accessibility of mainstream services and various models of integrated care. Economic evaluation of pilots may be useful as there is a potential for financial savings, given the high frequency of unplanned healthcare among people who use illicit opioids. ${ }^{35}$

\section{Twitter Dan Lewer @danlewer}

Acknowledgements Participants in this study voluntarily gave their time.

Contributors MB and DL planned the study, conducted the interviews, transcribed the data, conducted the analysis and wrote the manuscript. MB and DL read and approved the final manuscript.

Funding DL was funded by the National Institute for Health Research (NIHR) (Doctoral Research Fellowship DRF-2018-11-ST2-016). This paper presents independent research.

Disclaimer The views expressed are those of the authors and not necessarily those of the NHS, the NIHR or the Department of Health and Social Care.

Competing interests None declared.

Patient and public involvement Patients and/or the public were involved in the design, or conduct, or reporting, or dissemination plans of this research. Refer to the Methods section for further details.

Patient consent for publication Not required.

Ethics approval This study was approved by the UCL Research Ethics Committee on 1 April 2019 (Reference 13275/002).

Provenance and peer review Not commissioned; externally peer reviewed.

Data availability statement No data are available. Following the requirements of our ethical approval, interview transcripts will be deleted upon completion of the project.

Open access This is an open access article distributed in accordance with the Creative Commons Attribution Non Commercial (CC BY-NC 4.0) license, which permits others to distribute, remix, adapt, build upon this work non-commercially, and license their derivative works on different terms, provided the original work is properly cited, appropriate credit is given, any changes made indicated, and the use is non-commercial. See: http://creativecommons.org/licenses/by-nc/4.0/.

ORCID iDs

Molly Bradbury http://orcid.org/0000-0002-1894-1752

Dan Lewer http://orcid.org/0000-0003-3698-7196

\section{REFERENCES}

1 Degenhardt L, Bucello C, Mathers B, et al. Mortality among regular or dependent users of heroin and other opioids: a systematic review and meta-analysis of cohort studies. Addiction 2011;106:32-51.

2 Mathers BM, Degenhardt L, Bucello C, et al. Mortality among people who inject drugs: a systematic review and meta-analysis. Bull World Health Organ 2013;91:102-23.

3 Lewer D, Tweed EJ, Aldridge RW, et al. Causes of hospital admission and mortality among 6683 people who use heroin: a cohort study comparing relative and absolute risks. Drug Alcohol Depend 2019;204:107525. 
4 Degenhardt L, Larney S, Randall D, et al. Causes of death in a cohort treated for opioid dependence between 1985 and 2005. Addiction 2014;109:90-9.

5 Darke S, Lappin J, Farrell M. The Clinician's guide to illicit drugs and health. Bream: Silverback Publishing, 2019.

6 Public Health England. Substance misuse treatment for adults: statistics 2019 to 2020, 2018. Available: https://www.gov.uk/ government/statistics/substance-misuse-treatment-for-adultsstatistics-2019-to-2020 [Accessed 28 Jan 2020].

7 National Drug Evidence Centre. Drug treatment activity in England 2012-13, 2013. Available: https://webarchive.nationalarchives. gov.uk/20170807160711/http://www.nta.nhs.uk/statistics.aspx [Accessed 5 May 2021].

8 Neale J, Tompkins C, Sheard L. Barriers to accessing generic health and social care services: a qualitative study of injecting drug users. Health Soc Care Community 2008;16:147-54.

9 Ross LE, Vigod S, Wishart J, et al. Barriers and facilitators to primary care for people with mental health and/or substance use issues: a qualitative study. BMC Fam Pract 2015;16:135.

10 Weiss L, McCoy K, Kluger M, et al. Access to and use of health care: perceptions and experiences among people who use heroin and cocaine. Addict Res Theory 2004;12:155-65.

11 van Boekel LC, Brouwers EPM, van Weeghel J, et al. Stigma among health professionals towards patients with substance use disorders and its consequences for healthcare delivery: systematic review. Drug Alcohol Depend 2013;131:23-35.

12 Mistral W, Velleman R. Substance-misusing patients in primary care: incidence, services provided and problems. A survey of general practitioners in Wiltshire. Drugs 2001;8:61-72.

13 Jones HE, Harris RJ, Downing BC. Estimating the prevalence of problem drug use from drug-related mortality data. Addiction 2020:add.15111.

14 Kalk NJ, Robertson JR, Kidd B, et al. Treatment and intervention for opiate dependence in the United Kingdom: lessons from triumph and failure. Eur J Crim Policy Res 2018;24:183-200.

15 Mason T, Sutton M, Whittaker W, et al. The impact of paying treatment providers for outcomes: difference-in-differences analysis of the 'payment by results for drugs recovery' pilot. Addiction 2015;110:1120-8.

16 Mitchell CA, Pitt A, Hulin J, et al. Respiratory health screening for opiate misusers in a specialist community clinic: a mixed-methods pilot study, with integrated staff and service user feedback. BMJ Open 2016;6:e012823.

17 Burhan H, Young R, Byrne T, et al. Screening heroin smokers attending community drug services for COPD. Chest 2019;155:279-87.

18 Socías ME, Karamouzian M, Parent S, et al. Integrated models of care for people who inject drugs and live with hepatitis $C$ virus: a systematic review. Int J Drug Policy 2019;72:146-59.

19 EMCDDA. Hepatitis C: new models of care for drugs services, 2019 Available: http://www.emcdda.europa.eu/drugs-library/hepatitis-cnew-models-care-drugs-services [Accessed 12 May 2020]
20 Clinical Guidelines on Drug Misuse and Dependence Update 2017 Independent Expert Working Group. Drug misuse and dependence: UK guidelines on clinical management, 2017. Available: https:// assets.publishing.service.gov.uk/government/uploads/system/ uploads/attachment_data/file/673978/clinical_guidelines_2017.pdf [Accessed 29 Jan 2019].

21 Patton MQ. Qualitative Research \& Evaluation Methods. 4th edn. California: Sage, 2015.

22 Braun V, Clarke V. Using thematic analysis in psychology. Qual Res Psychol 2006;3:77-101.

23 Neale J, Tompkins C, Sheard L. Barriers to accessing generic health and social care services: a qualitative study of injecting drug users. Health Soc Care Community 2007;16:147-54.

24 Bramley G, Fitzpatrick S, Wood J. Hard edges Scotland, 2019. Available: https://lankellychase.org.uk/wp-content/uploads/2019/06/ Hard-Edges-Scotland-full-report-June-2019.pdf [Accessed 25 Feb 2020].

25 Advisory Council on the Misuse of Drugs. ACMD report: ageing cohort of drug users, 2019. Available: https://www.gov.uk/ government/publications/acmd-report-ageing-cohort-of-drug-users [Accessed 10 Oct 2019].

26 Rodgers C. The Kirketon Road Centre - improving access to primary care for marginalised populations. Aust Fam Physician 2012;41:245-7.

27 McGuire J, Gelberg L, Blue-Howells J, et al. Access to primary care for homeless veterans with serious mental illness or substance abuse: a follow-up evaluation of co-located primary care and homeless social services. Adm Policy Ment Health 2009;36:255-64.

28 Hoang T, Goetz MB, Yano EM, et al. The impact of integrated HIV care on patient health outcomes. Med Care 2009;47:560-7.

29 Islam MM, Topp L, Day CA, et al. The accessibility, acceptability, health impact and cost implications of primary healthcare outlets that target injecting drug users: a narrative synthesis of literature. Int $J$ Drug Policy 2012;23:94-102.

30 Friedmann PD, Zhang Z, Hendrickson J, et al. Effect of primary medical care on addiction and medical severity in substance abuse treatment programs. J Gen Intern Med 2003;18:1-8.

31 Weisner C, Mertens J, Parthasarathy S, et al. Integrating primary medical care with addiction treatment: a randomized controlled trial. JAMA 2001;286:1715-23.

32 European Monitoring Centre for Drugs and Drug Addiction. Drug consumption rooms: an overview of provision and evidence, 2018. Available: https://www.emcdda.europa.eu/publications/pods/drugconsumption-rooms_en [Accessed 12 May 2020].

33 Drummond C. Cuts to addiction services are a false economy. BMJ 2017;357:j2704.

34 Black C. Review of drugs: phase one report, 2020. Available: https:// www.gov.uk/government/publications/review-of-drugs-phase-onereport [Accessed 12 May 2020].

35 Lewer D, Freer J, King E, et al. Frequency of health-care utilization by adults who use illicit drugs: a systematic review and meta-analysis. Addiction 2020;115:1011-23. 